\title{
QUEEN'S
UNIVERSITY
BELFAST
}

\section{Higher aqueous levels of matrix metalloproteinases indicated visual impairment in patients with retina vein occlusion after anti-VEGF therapy}

Yong, L., Wan, J., Luo, C., Zhou, Y., Xu, H., \& Chen, Z. (2021). Higher aqueous levels of matrix metalloproteinases indicated visual impairment in patients with retina vein occlusion after anti-VEGF therapy. British Journal of Ophthalmology, 105(7), 1029-1034. https://doi.org/10.1136/bjophthalmol-2019-315358

Published in:

British Journal of Ophthalmology

\section{Document Version:}

Peer reviewed version

Queen's University Belfast - Research Portal:

Link to publication record in Queen's University Belfast Research Portal

\section{Publisher rights}

(c) Author(s) (or their employer(s)) 2019.

This manuscript is distributed under a Creative Commons Attribution-NonCommercial License (https://creativecommons.org/licenses/bync/4.0/), which permits use, distribution and reproduction for non-commercial purposes, provided the author and source are cited.

\section{General rights}

Copyright for the publications made accessible via the Queen's University Belfast Research Portal is retained by the author(s) and / or other copyright owners and it is a condition of accessing these publications that users recognise and abide by the legal requirements associated with these rights.

Take down policy

The Research Portal is Queen's institutional repository that provides access to Queen's research output. Every effort has been made to ensure that content in the Research Portal does not infringe any person's rights, or applicable UK laws. If you discover content in the

Research Portal that you believe breaches copyright or violates any law, please contact openaccess@qub.ac.uk. 
Higher aqueous levels of Matrix Metalloproteinases (MMPs) indicated visual impairment in patients with retina vein occlusion after anti-VEGF therapy

Yong Luo ${ }^{\mathrm{a}}$, Jianbo $\mathrm{Wan}^{\mathrm{b}}$, Chang Luo ${ }^{\mathrm{a}, \mathrm{c}}$, HengWei Liu ${ }^{\mathrm{a}}$, YuFan Zhou ${ }^{\mathrm{a}}$, Heping Xu ${ }^{\mathrm{a}, \mathrm{c}, \mathrm{d}^{*}}$ and ZhongPing Chen ${ }^{\mathrm{a}, \mathrm{b}^{*}}$

aAIER School of Ophthalmology, Central South University, Changsha 410015, Hunan Province, China;

${ }^{b}$ AIER Eye Hospital of Changsha, Changsha 410015, Hunan Province, China;

cAier Eye Institute, Changsha, Hunan, 410015, China;

d The Wellcome-Wolfson Institute for Experimental Medicine, School of Medicine, Dentistry and Biomedical Sciences, Queen' s University Belfast, UK.

*Corresponding author: Heping Xu; e-mail: heping.xu@qub.ac.uk;

ZhongPing Chen; e-mail: chenzhongping@aierchina.com 


\section{Synopsis}

Patients with retinal vein occlusion had higher intraocular levels of matrix metalloproteinase (MMP)-1, MM-2, MMP-7 and MMP-9. The intraocular levels of MMPs are negatively correlated with visual improvement in patients. 


\title{
Higher aqueous levels of Matrix Metalloproteinases (MMPs) indicated visual impairment in patients with retina vein occlusion after anti-VEGF therapy
}

\begin{abstract}
Purpose: To investigate the levels of matrix metalloproteinases (MMPs) in aqueous humor of patients with retinal vein occlusion (RVO) and the relationship between intraocular MMP levels and retinal lesion and visual prognosis.
\end{abstract}

Materials and Methods: 52 RVO patients, including 23 with central retinal vein occlusion (CRVO) and 29 with branch retinal vein occlusion (BRVO) and 20 participants with senile cataract were enrolled in this study. Retinal lesions were examined by fundus colour photography, fluorescein fundus angiography (FFA) and optical coherence tomographic angiography (OCTA). Sixty microliters of aqueous humor were collected during intravitreal anti-VEGF injection or cataract surgery. The aqueous levels of MMP1, MMP2, MMP7, MMP9 and MMP10 were measured using the Luminex xMAP Multiplex assay. The relationship between MMP levels and clinical presentations was analyzed by Pearson correlation test.

Results: The aqueous humor levels of MMP-1, MMP-2, MMP-7 and MMP-9, but not MMP10 in RVO patients were significantly higher than those in people with cataract after adjusting for age. Further analysis of RVO subgroups showed that the aqueous humor level of MMP2 in CRVO was significantly higher than that in BRVO. The aqueous humor levels of MMP-1 and MMP-2 were positively correlated with superficial capillary plexus vessel density (SVD); whereas the aqueous humor levels of MMP-1 and MMP-7 were negatively correlated with visual improvement following treatment. No correlation between aqueous humor levels of MMP and disease duration and central retinal thickness was observed. 
Conclusions: RVO eyes had significantly higher intraocular levels of MMP-1, MMP-2, MMP-7 and MMP-9 than cataract eyes and the level of MMP2 appears to be related to the area of occlusion. Intraocular levels of MMP may positively affect SVD and negatively impact visual function in RVO.

Keywords: retinal vein occlusion, matrix metalloproteinase, aqueous humour, visual prognosis, OCT angiography. 


\section{Introduction}

Retinal vein occlusion (RVO) is the second most common retinal vascular disease after diabetic retinopathy, with an estimated prevalence of more than 16 million worldwide and a common cause of vision loss(1) Retinal vein occlusion can be further divided into central (CRVO) or branch RVO (BRVO). The prevalence of CRVO is lower than that of BRVO (2). Due to the disturbance of retinal circulation and tissue hypoxia, serious complications such as macular edema, retinal hemorrhage, circuitous dilatation of blood vessels, neovascularization and fibrovascular tissue formation often occur in RVO patients. The mainstream therapies of RVO include intraocular injection of VEGF inhibitors, surgery and laser photocoagulation. Anti-VEGF therapy is effective in reducing macular edema and improving visual acuity (3-5). However, the visual outcome varies in patients. Current management of CRV is not satisfactory (6-9). The mechanism of RVO remains to be fully elucidated.

Matrix metalloproteinases (MMPs) are important mediators of vascular inflammation and remodeling (10-12). MMPs are known to be involved in the pathogenesis of cerebral hemorrhage and infarction the cerebral levels of MMP are closely related to disease progression (13). Furthermore, it has been reported that increased plasma MMP-9 level is an risk factor for hematoma dilatation (HE) in patients with acute hypertensive intracerebral hemorrhage (14). The severity of cerebral infarction in patients with acute myocardial infarction (AMI) complicated with cerebral infarction was positively correlated with the serum levels of MMP-2 and MMP-9(15). The increase in hydrostatic venous pressure in lower extremities can upregulate MMP expression, degradation of extracellular matrix (ECM), dysregulation of vasoconstriction and relaxation; ultimately, it may lead to venous thrombosis and thrombophlebitis $(16,17)$. 
Abnormal expression of MMP has been observed in retinal vascular diseases. For example, higher intraocular levels of MMP1 and MMP9 have been observed in diabetic retinopathy and higher levels of MMP2 and TIMP1/2 have been detected in the aqueous humour in high myopia patients $(18,19)$. In addition, increased levels of MMP9 have been reported in the vitreous humour in three RVO patients(8). The intraocular level of other MMPs in RVO has not been reported. In this study, we measured the levels of MMP-1, $-2,-7,-9$, and -10 in aqueous humor of 52 RVO patients (including 23 CRVO and 29 BRVO) and 20 cataract patients and examined the relationship between MMP levels and retinal lesions and visual improvement following treatment.

\section{Materials and methods}

\subsection{Patients}

This study was performed in accordance with the Declaration of Helsinki. Informed consent was obtained from each participant and the Institutional Review Board (IRB) at the Changsha Aier Eye Hospital approved the study (IRB number: 201605). 52 patients with RVO (23 with CRVO and 29 with BRVO) and 20 senile cataract patients were recruited to this prospective observational case-control study.

Inclusion criteria were RVO confirmed by fundus stereoscopy and fluorescein fundus angiography (FFA) after ruling out other retinal diseases. Exclusion criteria were the presence or history of intraocular or systemic inflammatory diseases, presence or history of cancer, autoimmune diseases, ocular trauma, liver and kidney dysfunction, previous intraocular surgery, or laser therapy within 6 months. The inclusion criteria of the control group were elderly cataract patients who needed phacoemulsification. Exclusion criteria were the same as above. 


\subsection{Clinical examination}

Comprehensive ophthalmic examinations were performed at baseline, including logMAR best corrected visual acuity (BCVA), slit lamp examination, intraocular pressure measurement and fundus stereoscopic biomicroscopy, fluorescein fundus angiography (FFA), OCT and OCTA. All patients participated in this study received intravitreal anti-VEGF (Ranibizumab or Conbercept) therapy and then were subjected to "injections as needed" (PRN) regime during monthly follow up visits. Patients who met any of the following criteria were treated again with additional intravitreal ranibizumab or conbercept: (1) no signs of improvement in the central retinal thickness (CRT) following previous treatment; (2) occurrence of additional lesion, e.g., retinal edema and hemorrhage. Monthly follow-up examinations included visual acuity, intraocular pressure, slit lamp examination, fundus biomicroscopy. Patients were followed up for 6-8 months. LogMAR visual acuity and CRT were recorded 6 to 8 months after the first treatment. The improvement of visual acuity was calculated by subtracting of LogMAR score of the final visit from the LogMAR score at the first visit (before treatment); whereas, the CRT changes were calculated by subtracting the CRT thickness at the final visit from the CRT thickness at the first visit.

\subsection{Sample collection}

Aqueous humor $(60 \mu \mathrm{L})$ was collected during the first intravitreal anti-VEGF injection or during cataract surgery and the procedure was conducted in an Ophthalmology surgical operating room. All procedures strictly followed the aseptic operating principles of internal eye surgery. A puncture was made at the limbus and $60 \mu \mathrm{L}$ of aqueous humor was drawn from the anterior chamber using disposable sterile syringe and then transferred into a sterile Eppendorf and stored at $-80^{\circ} \mathrm{C}$ until measurement of MMPs. 


\subsection{Measurement of MMPs}

The levels of MMP-1, MMP-2, MMP-7, MMP-9 and MMP-10 in aqueous humor samples were measured using the MILLIPLEX Miltiplex assay following manufacture' s instructions (Luminex xMap Technology, Bio-Rad, Hercules, CA, USA). A total of 20 uL of aqueous humor from each sample (1:2 dilution) was used in the study. The Median Fluorescent Intensity (MFI) in each well in the plates were measured using a plate reader with the MAGPIX ${ }^{\circledR}$ with $x$ PONENT software (Millipore Technology, Hercules, CA, USA). The MMP concentrations in samples were calculated using the spline curve-fitting method.

\subsection{Statistics}

Data were analyzed using the SPSS 20.0 software. The normal distribution of continuous variables was evaluated by KolmogorovSmirnov test, and the continuous samples of normal distribution were compared by independent sample $t$ test or one-way ANOVA. Chi-square test and independent sample t-test were used to compare the general clinical data. For the meaningful correlation in univariate analysis, covariance analysis is carried out to adjust the parameters. The relationship between MMP levels and clinical parameters was analyzed using the Pearson correlation test. $\mathrm{P}<0.05$ was considered statistically significant.

\section{Results:}

\subsection{Clinical characteristics}

The clinical data of the participants in each group are shown in Table 1. There was no significant difference in sex distribution, history of hypertension, history of diabetes, body mass index, smoking habit and initial visual acuity (before treatment) between RVO patients and cataract controls. However, the average age of RVO patients (59.71 \pm 11.90$)$ was significantly younger than controls $(67.35 \pm 6.98)$. The visual acuity (LogMAR) 
of RVO patients at the final visit $(0.94 \pm 0.62)$ was significantly worse than that of the cataract control group (0.24 \pm 0.23$)$. The initial CRT of RVO patients $(660.31 \pm 255.07)$ was significantly higher than that in Cataract patients $(216.45 \pm 10.06)$ (Table 1).

Within the RVO patients, the age, sex distribution, history of hypertension, history of diabetes, body mass index and smoking habit did not differ between BRVO and CRVO. The visual acuities (LogMAR) of CRVO patients at the first visit $(1.35 \pm 0.62)$ and final visit $(1.27 \pm 0.70)$ were significantly worse than BRVO patients $(0.93 \pm 0.43$ at first visit and $0.68 \pm 0.39$ at final visit, respectively). CRVO patients also had significantly higher CRT at the first visit $(796.52 \pm 251.91)$ and final visit $(344.35 \pm 136.37)$ than BRVO patients $(552.28 \pm 203.26$ at initial visit, $274.97 \pm 80.70$ at final visit) (Table 1).

\subsection{MMPs level in aqueous humor of RVO patients and controls}

The levels of MMP1, MMP-2, MMP-7 and MMP-9 in the RVO group were significantly higher than that in the control group (Table 2). The level of MMP-10 did not differ significantly between the two groups (Table 2).

Further subgroup analysis showed that the levels of MMP-1, MMP-2, MMP-7, MMP-9 in CRVO patients were significantly higher than those in controls (Table 3). The differences remained significant after adjusting for age (Table3). The levels of MMP-2 in BRVO patients was also significantly higher than that in controls $(\mathrm{P}=$ 0.006). Furthermore, patients with CRVO had significantly higher levels of MMP2 than patients with BRVO even after adjusting for age $(P<0.048$, Table 3), suggesting that the aqueous humor level of MMP2 is related to the area of RVO.

\subsection{Correlation analysis of OCTA parameters and MMPs in aqueous humor of RVO patients}

Of the 52 RVO patients, 17 had OCTA examination before treatment. Pearson correlation analysis was 
conducted to evaluate possible link between aqueous levels of MMPs and RVO-mediated retinal structural alteration. MMP-1 was positively correlated with ILM-IPL thickness $(r=0.481, P=0.05)$ and superficial capillary plexus vessel density (SVD, $r=0.610, P=0.009)$. MMP-2 was also positively correlated with SVD $(r=$ $0.560, \mathrm{P}=0.019$ ) in RVO patients (Table 4). No correlation was observed between the aqueous humor levels of MMP and the size of foveal avascular zone, perimeter of foveal avascular zone (PERIM), central retinal thickness, the vessel density of deep capillary plexus and initial visual acuity (Table 4).

\subsection{Correlation between MMP concentrations and disease duration and visual improvement in RVO}

\section{patients}

There was no significant correlation between the duration of RVO (i.e. from disease onset to aqueous humor sample collection) and any of the MMPs. All RVO patients were followed up for at least 6 months and the visual improvement negatively correlated with the aqueous humor levels of MMP-1 $(r=-0.385, p=0.005)$, MMP-7 $(r=-0.319, p=0.021)$ and change of CRT $(r=-0.332, p=0.016)$. There was no correlation between the aqueous humor levels of MMP-2, MMP-9, MMP-10 and visual improvement (Table 5).

\section{Discussion:}

In this study, we show that the aqueous levels of MMP1, MMP2, MMP7 and MMP9 were significantly increased in RVO patients compared with cataract controls. In general, the aqueous levels of MMP were higher in CRVO than those in BRVO, but none of the MMPs correlated with initial CRT and visual acuity suggesting that the aqueous humor levels of MMP is related to the area of retinal vein occlusion but not the severity of macular lesion. We further found that aqueous levels of MMP, in particular, MMP1 is positively correlated with retinal superficial capillary plexus vessel density but negatively correlated with visual 
improvement. Our results suggest that intraocular levels of MMP may be critically involved in retinal pathology in RVO.

Retinal hemorrhage, macular edema and neovascularization are the common cause of visual impairment in RVO. Retinal vein thrombosis disrupts upstream blood circulation leading to interstitial fluid and protein accumulation, microcirculatory ischemia and tissue edema $(20,21)$. The inflammatory response induced by vascular occlusion further damages retinal vasculature and neurons and accelerates disease progression (22). MMPs are a class of zinc-dependent proteases that are involved in the degradation of ECM proteins. There are 28 members of the MMP family, of which 14 are expressed in human vascular tissues and cells, including vascular smooth muscle (VSM), fibroblasts and leukocytes (23). Based on their enzymatic activities, MMP can be divided into collagenase, gelatinase, matrix lyase and matrix protease, etc. Activation of different MMPs can lead to the degradation of collagen, elastin and other ECM proteins, respectively, that may severely affect vascular function. In the venous vessel wall, MMPs are expressed in different layers, including adventitia, media and intima, and may interact with ECM, VSM and endothelial cells. Previous studies have shown that MMP-1 and-7 are specifically expressed by vascular endothelial cells and VSM cells $(24,25)$. The vascular endothelial cells have a complex enzyme system that can synthesize and secrete a variety of bioactive substances. The intima composed of vascular endothelial cells forms a permeability barrier, which enables liquids, gases and macromolecular substances to selectively pass through the vessel. In addition, the media composed of elastic fibers and VSM can contract and dilate blood vessels and the collagen fibers play an important role in maintaining vascular tension. Degradation of the collagen fibers by MMPs may severely affect vascular stability and elasticity. 
In this study, we found that the aqueous humor levels of MMP1, MMP2, MMP7 and MMP9 were significantly higher in CRVO patients compared with those in cataract patients even after correction for age. A previous by Tuuminen et al reported higher aqueous humor levels of MMP-2 and MMP-9 in RVO patients (8), which is line with our observation. MMP1 is a key collagenase and MMP2 and MMP9 are gelatinases. MMP7 can degrade fibronectin and laminin. Activation of these MMPs inside the eye would affect the stability and elasticity of retinal vasculature, therefore, may directly contribute to CRVO pathologies.

In this small case study, the CRT in CRVO patients was higher than that in BRVO patients at the initial visit, suggesting more severe macular oedema in CRVO patients in general. Interestingly, the changes (reductions) in CRT were negatively correlated with visual improvement at the final visit. This result suggests that severe macular oedema in CRVO may cause irreversible visual damage and the reduction in macular oedema following anti-VEGF therapy may not always improve vision.

In our study, the aqueous humor levels of MMP-1 and MMP-7 were negatively correlated with visual improvement. This may be related to their enzymatic activity to collagen and laminin/fibronectin, which may lead to vascular leakage, inflammation, macular oedema and visual impairment (26). Compared with BRVO patients, CRVO patients had a wider range of embolic areas, more severe retinal ischemia and hypoxia, and higher aqueous humor levels of MMPs, suggesting that aqueous humor levels of MMPs might be related to the area of vascular occlusion. Our results suggest that the aqueous humor level of MMP-1 and MMP-7 may be useful biomarkers for RVO prognosis. They may also be targeted for RVO management.

In this study, MMP-1 and MMP-2 were positively correlated with macular superficial capillary density. Winegarner et al reported a positive correlation between better BCVA and superficial capillary 
density in RVO patients (27); whereas we observed a negative correlation between MMP1 and visual improvement. In our study, the aqueous humor level of MMP1, superficial capillary density and visual improvement do not support each other. This may be due to the small sample size and variations in clinical presentation. Our research has a few limitations. First, we have relatively small sample size, especially only a few patients underwent OCTA examination. Second, although RVO patients were followed up for 6 to 8 months, MMP levels were only measured at the basal level i.e., prior to treatment. We, therefore, were unable to determine the dynamic change of MMP during disease progression. Third, this study was conducted in a single-center and results only reflect the biological feature of RVO in local ethical population. Further multicenter study will be needed to verify the results in other populations.

In conclusion, we show that RVO patients had higher intraocular levels of MMP-1, MMP-2, MMP-7 and MMP-9 and the levels of MMP appear to be related to the area of retinal vascular occlusion. Intraocular levels of MMP may positively affect SVD and negatively impact visual function in RVO. The aqueous humor levels of MMP1 and MMP7 may be useful biomarkers for RVO prognosis. Our study highlights the needs for further investigation in the role of MMPs in RVO initiation and progression.

\section{Declaration of interest}

All authors have no financial interest in the publication of this article.

\section{Funding}

This work was supported by the Natural Science Foundation Project of Hunan Province [2018JJ2001], the Research Fund Project of AIER Eye Hospital Group [AF1601D5 and AM1901D5], Hunan Science \& Technology Association [2018KX001], Science \& Technology 
Department of Human Province [2018RS3123] and Overseas Talent \& Intelligence Project of Changsha City.

\section{Acknowledgements}

The authors thank the staff at the Changsha Aier Eye Hospital and Aier Eye Institute for their assistance in this research. 


\section{References}

1. Ho M, Liu DTL, Lam DSC, Jonas JB. RETINAL VEIN OCCLUSIONS, FROM BASICS TO THE LATEST TREATMENT. Retina. 2016.

2. Rogers S, Mcintosh RL, Ning C, Lim L, Jie JW, Mitchell P, et al. The Prevalence of Retinal Vein Occlusion: Pooled Data from Population Studies from the United States, Europe, Asia, and Australia. Ophthalmology. 2010;117(2):313-9.e1.

3. Campochiaro PA, Heier JS, Feiner L, Gray S, Rubio RG. Ranibizumab for Macular Edema following Branch Retinal Vein Occlusion:: Six-Month Primary End Point Results of a Phase III Study. Ophthalmology. 2010;117(6):1102-12.e1.

4. DM B, PA C, RP S, Z L, S G, N S, et al. Ranibizumab for macular edema following central retinal vein occlusion: six-month primary end point results of a phase III study. Ophthalmology. 2010;117(6):1124-33.e1.

5. M I, TA M, G H, I Z-G, AW S, SD S, et al. Loss of Peak Vision in Retinal Vein Occlusion Patients Treated for Macular Edema. American journal of ophthalmology. 2019;205(undefined):17-26.

6. Sveinn Hakon H, Einar S. Oxygen saturation in central retinal vein occlusion. American Journal of Ophthalmology. 2010;150(6):871-5.

7. Laatikainen L, Kohner EM. Fluorescein angiography and its prognostic significance in central retinal vein occlusion. Br J Ophthalmol. 1976;60(6):411-8.

8. Tuuminen R, Loukovaara S. High intravitreal TGF- $\beta 1$ and MMP-9 levels in eyes with retinal vein occlusion. Eye. 2014;28(9):1095-9.

9. Nishiyama Y. Retinal oxygen saturation levels in patients with central retinal vein occlusion. Ophthalmology. 2002;109(8):1521-6.

10. $X Z, W J A, P T, A F D, R$ G. Role of matrix metalloproteinases in the pathogenesis of intracranial aneurysms. Neurosurgical focus. 2019;47(1):E4.

11. VA M, DA C, AV G, AN O. Matrix metalloproteinases in pro-atherosclerotic arterial remodeling. Journal of molecular and cellular cardiology. 2018;123(undefined):159-67.

12. LN T, EJ H, M C, K N, SY L, JR P, et al. Distinct Roles for Matrix Metalloproteinases 2 and 9 in Embryonic Hematopoietic Stem Cell Emergence, Migration, and Niche Colonization. Stem cell reports. 2017;8(5):1226-41.

13. Dang B, Duan X, Wang Z, He W, Chen G. A therapeutic target of cerebral hemorrhagic stroke: matrix metalloproteinase-9. Current Drug Targets. 2017;18(999):1-.

14. Qingwei Y, Xiaorong Z, Feng P, Weihong Z. Relationship of plasma matrix metalloproteinase-9 and?hematoma expansion in acute hypertensive cerebral hemorrhage. International Journal of Neuroscience. 2016;126(3):213-8.

15. Zhou L, Kou DQ. Correlation between acute myocardial infarction complicated with cerebral infarction and expression levels of MMP-2 and MMP-9. Eur Rev Med Pharmacol Sci. 2019;23(1):297-302.

16. MacColl E, Khalil RA. Matrix Metalloproteinases as Regulators of Vein Structure and Function: Implications in Chronic Venous Disease. The Journal of pharmacology and experimental therapeutics. 2015;355(3):410-28.

17. Kucukguven A, Khalil RA. Matrix metalloproteinases as potential targets in the venous dilation associated with varicose veins. Curr Drug Targets. 2013;14(3):287-324.

18. Kwon JW, Choi JA, Jee D. Matrix Metalloproteinase-1 and Matrix Metalloproteinase-9 in the Aqueous Humor of Diabetic Macular Edema Patients. PloS one. 2016;11(7):e0159720.

19. Jia Y, Hu DN, Sun J, Zhou J. Correlations Between MMPs and TIMPs Levels in Aqueous Humor from High Myopia and Cataract Patients. Current eye research. 2017;42(4):600-3. 
20. Karia N. Retinal vein occlusion: pathophysiology and treatment options. Clinical ophthalmology (Auckland, NZ). 2010;4:809-16.

21. Akitaka T, Atsushi S, Masafumi O, Yuriko K, Hideyasu O, Kazuaki M, et al. Serous retinal detachment associated with retinal vein occlusion. American Journal of Ophthalmology. 2010;149(2):291-301.e5.

22. Noma H, Yasuda K, Shimura M. Cytokines and the Pathogenesis of Macular Edema in Branch Retinal Vein Occlusion. Journal of ophthalmology. 2019;2019:5185128.

23. Robert V, Hideaki N. Matrix metalloproteinases and tissue inhibitors of metalloproteinases: structure, function, and biochemistry. Circulation Research. 2003;92(8):827-39.

24. Sansilvestri-Morel P, Fioretti F, Rupin A, Senni K, Fabiani JN, Godeau G, et al. Comparison of extracellular matrix in skin and saphenous veins from patients with varicose veins: does the skin reflect venous matrix changes? Clinical Science. 2007;112(4):229-39.

25. Cui N, Hu M, Khalil RA. Biochemical and Biological Attributes of Matrix Metalloproteinases. Progress in Molecular Biology \& Translational Science. 2017;147:1.

26. Webster L, Chignell AH, Limb GA. Predominance of MMP-1 and MMP-2 in Epiretinal and Subretinal Membranes of Proliferative Vitreoretinopathy. Experimental Eye Research. 1999;68(1):91-8.

27. Winegarner A, Wakabayashi T, Fukushima Y, Sato T, Hara-Ueno C, Busch C, et al. Changes in Retinal Microvasculature and Visual Acuity After Antivascular Endothelial Growth Factor Therapy in Retinal Vein Occlusion. Invest Ophthalmol Vis Sci. 2018;59(7):2708-. 
Table1.Basic Characteristics and Clinical Data of the Study Subjects

\begin{tabular}{|c|c|c|c|c|c|c|}
\hline \multirow{3}{*}{ General information } & \multirow{3}{*}{ RVO $(n=52)$} & \multirow{3}{*}{ BRVO $(n=29)$} & \multirow{3}{*}{$\begin{array}{l}\text { CRVO } \\
(n=23)\end{array}$} & \multirow{3}{*}{ Control $(n=20)$} & \multirow{2}{*}{$\begin{array}{l}\text { P-values } \\
\text { Controls vs }\end{array}$} & \multirow{2}{*}{$\begin{array}{l}\text { P-values } \\
\text { BRVO vs }\end{array}$} \\
\hline & & & & & & \\
\hline & & & & & RVO & CRVO \\
\hline Age (years) & $59.71 \pm 11.90$ & $58.76 \pm 11.62$ & $60.91 \pm 12.40$ & $67.35 \pm 5.98$ & $0.001^{a}$ & 0.522 a \\
\hline Female (number (\%)) & $21(40)$ & $11(38)$ & $10(43)$ & $11(55)$ & $0.264^{b}$ & $0.686^{b}$ \\
\hline \multicolumn{7}{|l|}{ Hypertension (number } \\
\hline & $30(58)$ & 17 (59) & $13(57)$ & $10(50)$ & $0.556^{b}$ & $0.879 \mathrm{~b}$ \\
\hline \multicolumn{7}{|l|}{ (\%)) } \\
\hline Diabetes (number (\%)) & $7(13)$ & $6(21)$ & $1(4)$ & $6(30)$ & $0.196^{b}$ & $0.117^{b}$ \\
\hline Smoking (number (\%)) & $8(15)$ & $4(14)$ & $4(17)$ & $5(25)$ & $0.543^{b}$ & $0.721^{b}$ \\
\hline Body mass index $(\mathrm{kg} / \mathrm{m} 2)$ & $24.88 \pm 3.10$ & $25.58 \pm 3.22$ & $24.01 \pm 2.78$ & $24.47 \pm 3.04$ & $0.609^{a}$ & $0.07^{a}$ \\
\hline Initial VA (LogMAR) & $1.12 \pm 0.56$ & $0.93 \pm 0.43$ & $1.35 \pm 0.62$ & $0.98 \pm 0.58$ & $0.347^{a}$ & 0.009 a \\
\hline Final visit VA (LogMAR) & $0.94 \pm 0.62$ & $0.68 \pm 0.39$ & $1.27 \pm 0.70$ & $0.24 \pm 0.23$ & $<0.001^{a}$ & $0.001^{a}$ \\
\hline Initial CRT & $660.31 \pm 255.07$ & $552.28 \pm 203.26$ & $796.52 \pm 251.91$ & $216.45 \pm 10.06$ & $<0.001^{a}$ & $<0.001^{a}$ \\
\hline Final visit CRT & $305.65 \pm 113.18$ & $274.97 \pm 80.70$ & $344.35 \pm 136.37$ & / & / & $0.038{ }^{a}$ \\
\hline
\end{tabular}

Values are given as Mean \pm SD $(n=72)$.

Initial VA= Initial visual acuity, Final visit VA= Final visit visual acuity; Initial CRT= Initial central retinal thickness; Final visit $C R T=$ Final visit central retinal thickness

In row, age and body mass index using independent sample T-test. Gender, hypertension, diabetes and smoking history using Chi-Square test. alndependent samples T-test . bPearson' s Chi-Square test.

Bold $\mathrm{P}<0.05$; / data not available 
Table2. Aqueous MMPs levels in RVO patients and controls

\begin{tabular}{llll}
\hline Variables & RVO $(\mathrm{n}=52, \mathrm{ng} / \mathrm{mL})$ & Control $(\mathrm{n}=20, \mathrm{ng} / \mathrm{mL})$ & P-values \\
\hline MMP-1 & $84.57 \pm 118.48$ & $4.59 \pm 5.86$ & $<\mathbf{0 . 0 0 1}$ \\
MMP-2 & $9392.71 \pm 5108.84$ & $4054.18 \pm 1955.52$ & $<\mathbf{0 . 0 0 1}$ \\
MMP-7 & $730.66 \pm 674.11$ & $339.81 \pm 230.05$ & $<\mathbf{0 . 0 0 1}$ \\
MMP-9 & $248.77 \pm 275.54$ & $92.94 \pm 102.07$ & $\mathbf{0 . 0 1 7}$ \\
MMP-10 & $328.55 \pm 694.44$ & $119.2 \pm 48.66$ & 0.184 \\
\hline
\end{tabular}

Values are given as Mean $\pm S D(n=72)$.

In row, using independent sample T-test.

Bold $\mathrm{P}<0.05$. 
Table3. Aqueous levels of MMP in CRVO and BRVO patients

\begin{tabular}{|c|c|c|c|c|c|c|c|}
\hline $\begin{array}{l}\text { MMPs } \\
(\mathrm{ng} / \mathrm{mL})\end{array}$ & $\begin{array}{l}\text { BRVO } \\
(\text { Mean } \pm S D) \\
n=29\end{array}$ & $\begin{array}{l}\text { CRVO } \\
(\text { Mean } \pm S D) \\
n=23\end{array}$ & $\begin{array}{l}\text { Control } \\
(\text { Mean } \pm S D) \\
n=20\end{array}$ & P-valuesa & $\begin{array}{l}\text { P-value } \\
\text { Bonferroni } \\
\text { post hoc test }\end{array}$ & P-valuesb & $\begin{array}{l}\text { P-value } \\
\text { Bonferroni } \\
\text { post hoc test }\end{array}$ \\
\hline \multirow[t]{3}{*}{ MMP-1 } & $68.54 \pm 89.41$ & $104.78 \pm 146.98$ & $4.59 \pm 5.86$ & 0.007 & $0.097 c$ & 0.014 & $0.192^{c}$ \\
\hline & & & & & $0.005^{d}$ & & $0.011^{d}$ \\
\hline & & & & & $0.605^{e}$ & & $0.567 \mathrm{e}$ \\
\hline \multirow[t]{3}{*}{ MMP-2 } & $8129.97 \pm 2875.68$ & $10984.87 \pm 6725.19$ & $4054.18 \pm 1955.52$ & $<0.001$ & $0.006^{c}$ & $<0.001$ & $0.028 c$ \\
\hline & & & & & $<0.001^{d}$ & & $<0.001^{d}$ \\
\hline & & & & & $0.064 \mathrm{e}$ & & $0.048 \mathrm{e}$ \\
\hline \multirow[t]{3}{*}{ MMP-7 } & $594.09 \pm 462.75$ & $902.84 \pm 851.65$ & $339.81 \pm 230.05$ & 0.008 & $0.402^{c}$ & 0.011 & $0.513^{c}$ \\
\hline & & & & & $0.006^{d}$ & & $0.01^{d}$ \\
\hline & & & & & $0.178^{e}$ & & $0.182^{\mathrm{e}}$ \\
\hline \multirow[t]{3}{*}{ MMP-9 } & $215.08 \pm 332.51$ & $291.24 \pm 178.64$ & $92.94 \pm 102.07$ & 0.031 & $0.256 c$ & 0.009 & $0.073^{c}$ \\
\hline & & & & & $0.026^{d}$ & & $0.008^{d}$ \\
\hline & & & & & $0.783^{e}$ & & $0.987 e$ \\
\hline \multirow[t]{3}{*}{ MMP-10 } & $396.24 \pm 923.31$ & $243.19 \pm 138.83$ & $119.2 \pm 48.66$ & 0.273 & $0.339 c$ & 0.085 & $0.083^{c}$ \\
\hline & & & & & $1.000^{d}$ & & $0.721^{d}$ \\
\hline & & & & & $1.000^{e}$ & & $0.784 \mathrm{e}$ \\
\hline
\end{tabular}

Values are given as SD standard deviation $(n=72)$

a, One-way ANOVA

b, univariate analysis corrected for age

c, Controls vs BRVO

d, Controls vs CRVO

e, BRVO vs CRVO 
Table 4. Correlation between Visual and OCTA parameters and MMPs RVO patients $(n=17)$

\begin{tabular}{|c|c|c|c|c|c|c|c|c|c|c|c|c|}
\hline Variable & MMP1 & MMP2 & MMP7 & MMP9 & MMP10 & FAZ & PERIM & $\mathrm{CRT}, \mu \mathrm{m}$ & ILM-IPL & SVD & DVD & $\begin{array}{l}\text { Initial } \\
\text { VA }\end{array}$ \\
\hline MMP1 & / & $0.538^{*}$ & $0.881^{\star \star}$ & 0.183 & -0.005 & -0.443 & -0.254 & 0.234 & 0.481 & $0.610^{\star *}$ & -0.018 & 0.053 \\
\hline MMP2 & 0.026 & / & 0.403 & 0.073 & -0.074 & -0.311 & -0.39 & 0.083 & 0.063 & $0.560^{*}$ & -0.037 & 0.306 \\
\hline MMP7 & $<0.001$ & 0.109 & / & $0.493^{*}$ & 0.324 & -0.332 & -0.089 & 0.161 & 0.399 & 0.459 & 0.016 & -0.049 \\
\hline MMP9 & 0.483 & 0.779 & 0.044 & / & $0.960 * *$ & -0.096 & 0.095 & 0.101 & 0.107 & 0.134 & -0.16 & 0.089 \\
\hline MMP10 & 0.985 & 0.777 & 0.205 & $<0.001$ & / & -0.023 & 0.158 & 0.033 & -0.024 & 0.026 & -0.135 & -0.062 \\
\hline FAZ & 0.075 & 0.225 & 0.193 & 0.715 & 0.929 & / & $0.836^{\star *}$ & -0.395 & -0.369 & $-0.601^{*}$ & -0.257 & -0.128 \\
\hline PERIM & 0.325 & 0.122 & 0.734 & 0.717 & 0.545 & $<0.001$ & / & -0.472 & -0.428 & $-0.587^{*}$ & -0.331 & -0.239 \\
\hline $\mathrm{CRT}, \mu \mathrm{m}$ & 0.367 & 0.75 & 0.537 & 0.699 & 0.9 & 0.116 & 0.056 & / & $0.849^{* *}$ & $0.737^{\star *}$ & 0.457 & 0.111 \\
\hline ILM-IPL & 0.05 & 0.811 & 0.113 & 0.683 & 0.928 & 0.144 & 0.086 & $<0.001$ & / & $0.597^{*}$ & 0.374 & 0.187 \\
\hline SVD & 0.009 & 0.019 & 0.064 & 0.608 & 0.92 & 0.011 & 0.013 & 0.001 & 0.011 & / & 0.227 & 0.163 \\
\hline DVD & 0.945 & 0.887 & 0.95 & 0.54 & 0.606 & 0.319 & 0.194 & 0.065 & 0.14 & 0.381 & / & -0.264 \\
\hline $\begin{array}{l}\text { Initial } \\
\text { VA }\end{array}$ & 0.839 & 0.232 & 0.852 & 0.734 & 0.813 & 0.623 & 0.355 & 0.671 & 0.472 & 0.532 & 0.323 & / \\
\hline
\end{tabular}

The value above the diagonal empty cell (/) represents the Pearson correlation coefficient

The value below the diagonal empty cell $(/)$ indicates the level of statistical significance of the correlation.

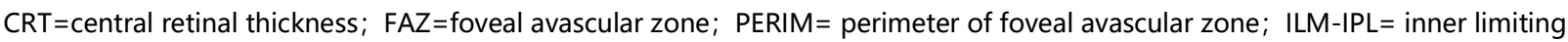
membrane-inner plexiform layer; SVD=superficial capillary plexus vessel density; DVD=deep capillary plexus vessel density; Initial $\mathrm{VA}=$ Initial visual acuity

${ }^{*} P \leq 0.05,{ }^{* *} P \leq 0.01$ 
Table 5. Correlation between MMPs and clinical parameters in RVO patients $(n=52)$

\begin{tabular}{llllllllll}
\hline Variable & MMP1 & MMP2 & MMP7 & MMP9 & MMP10 & RVO duration & Visual improvement & Change in CRT \\
\hline MMP1 & $/$ & $0.472^{* *}$ & $0.569^{* *}$ & 0.126 & 0.025 & -0.097 & $-0.385^{* *}$ & 0.089 \\
MMP2 & $<0.001$ & $/$ & $0.488^{* *}$ & 0.115 & 0.022 & 0.013 & -0.165 & 0.011 \\
MMP7 & $<0.001$ & $<0.001$ & $/$ & $0.487^{* *}$ & $0.325^{*}$ & -0.086 & $-0.319^{*}$ & 0.153 \\
MMP9 & 0.374 & 0.416 & $<0.001$ & $/$ & $0.859^{* *}$ & -0.029 & -0.177 & 0.193 \\
MMP10 & 0.86 & 0.877 & 0.019 & $<0.001$ & $/$ & -0.036 & -0.07 & 0.056 \\
RVO duration & 0.493 & 0.928 & 0.542 & 0.84 & 0.798 & $/$ & -0.113 & -0.034 \\
Visual improvement & 0.005 & 0.241 & 0.021 & 0.209 & 0.623 & 0.426 & $/$ & $-0.332^{*}$ \\
Change in CRT & 0.529 & 0.940 & 0.279 & 0.171 & 0.696 & 0.812 & 0.016 & $/$ \\
\hline
\end{tabular}

The value above the diagonal empty cell (/) represents the Pearson correlation coefficient ;

The value below the diagonal empty cell (/) indicates the level of statistical significance of the correlation ;

Change in CRT = Change in central retinal thickness ;

${ }^{*} \mathrm{P} \leq 0.05,{ }^{* *} \mathrm{P} \leq 0.01$ 